\title{
In Situ Surfactant Effects on Polymer/Reduced Graphene Oxide Nanocomposite Films: Implications for Coating and Biomedical Applications
}

Bich Ngoc Tran, ${ }^{1}$ Saroj Bhattacharyya,${ }^{2}$ Yin Yao, ${ }^{2}$ Vipul Agarwal, ${ }^{1, *}$ Per B. Zetterlund ${ }^{1, *}$

${ }^{1}$ Cluster for Advanced Macromolecular Design (CAMD), School of Chemical Engineering, University of New South Wales, Sydney, NSW 2052, Australia

${ }^{2}$ Mark Wainwright Analytical Centre, University of New South Wales, Sydney, New South Wales 2052, Australia

*Corresponding authors: agarwalvipu184@gmail.com; p.zetterlund@unsw.edu.au

\section{Supporting Information}

Table S1. Polymerization recipe for PSt/GO fabricated via miniemulsion polymerization at $70{ }^{\circ} \mathrm{C}$ after $24 \mathrm{~h}$ with $5 \mathrm{wt} \% \mathrm{GO}$ (rel. to St) using different surfactants (1 wt \% rel. to the organic phase).

\begin{tabular}{|c|c|c|c|c|c|c|}
\hline \multirow{2}{*}{ Monomer $^{\mathrm{a}}$} & \multirow{2}{*}{$\mathrm{HD}^{\mathrm{b}}$} & \multirow{2}{*}{$\mathrm{GO}^{\mathrm{b}}$} & \multirow{2}{*}{$\mathrm{AIBN}^{\mathrm{c}}$} & \multirow{2}{*}{ SDS $^{\mathrm{d}}$} & \multicolumn{2}{|c|}{ In situ surfactant } \\
\cline { 6 - 7 } & & & & & Acid $^{\mathrm{d}}$ & $\mathrm{KOH}^{\mathrm{e}}$ \\
\hline 7 & 5 & 5 & $0.25 \mathrm{M}$ & - & $1 \mathrm{SA}$ & 1.2 \\
\hline 7 & 5 & 5 & $0.25 \mathrm{M}$ & - & $1 \mathrm{OA}$ & 1.2 \\
\hline 7 & 5 & 5 & $0.25 \mathrm{M}$ & 1 & - & - \\
\hline
\end{tabular}

${ }^{\mathrm{a}} \mathrm{wt} \%$ rel. to water

${ }^{\mathrm{b}} \mathrm{wt} \%$ rel. to monomer

${ }^{c}$ Concentration rel. to monomer + HD

${ }^{\mathrm{d}} \mathrm{wt} \%$ rel. to organic phase

${ }^{\mathrm{e}} \mathrm{mol} \%$ rel. to acid

Table S2. Polymerization recipe for $\mathrm{P}(\mathrm{St}-$ stat- $n \mathrm{BA}) / \mathrm{GO}$ fabricated via miniemulsion polymerization at $70{ }^{\circ} \mathrm{C}$ after $24 \mathrm{~h}$ with $5 \mathrm{wt} \% \mathrm{GO}$ (rel. to monomer) using different surfactants.

\begin{tabular}{|c|c|c|c|c|c|c|}
\hline \multirow{2}{*}{ Monomer $^{\mathrm{a}}$} & \multirow{2}{*}{$\mathrm{HD}^{\mathrm{b}}$} & \multirow{2}{*}{$\mathrm{GO}^{\mathrm{b}}$} & \multirow{2}{*}{$\mathrm{AIBN}^{\mathrm{c}}$} & \multirow{2}{*}{$\mathrm{SDS}^{\mathrm{d}}$} & \multicolumn{2}{|c|}{ In situ surfactant } \\
\cline { 5 - 7 } & & & & & Acid $^{\mathrm{d}}$ & $\mathrm{KOH}^{\mathrm{e}}$ \\
\hline 7 & 5 & 5 & $0.25 \mathrm{M}$ & - & $1 \mathrm{SA}$ & 1.2 \\
\hline 7 & 5 & 5 & $0.25 \mathrm{M}$ & - & $3 \mathrm{SA}$ & 1.2 \\
\hline 7 & 5 & 5 & $0.25 \mathrm{M}$ & - & $5 \mathrm{SA}$ & 1.2 \\
\hline 7 & 5 & 5 & $0.25 \mathrm{M}$ & - & $7 \mathrm{SA}$ & 1.2 \\
\hline 7 & 5 & 5 & $0.25 \mathrm{M}$ & - & $1 \mathrm{OA}$ & 1.2 \\
\hline 7 & 5 & 5 & $0.25 \mathrm{M}$ & - & $3 \mathrm{OA}$ & 1.2 \\
\hline 7 & 5 & 5 & $0.25 \mathrm{M}$ & - & $5 \mathrm{OA}$ & 1.2 \\
\hline 7 & 5 & 5 & $0.25 \mathrm{M}$ & - & $7 \mathrm{OA}$ & 1.2 \\
\hline 7 & 5 & 5 & $0.25 \mathrm{M}$ & 1 & - & - \\
\hline 7 & 5 & 5 & $0.25 \mathrm{M}$ & 3 & - & - \\
\hline
\end{tabular}




\begin{tabular}{|l|l|l|l|l|l|l|}
\hline 7 & 5 & 5 & $0.25 \mathrm{M}$ & 5 & - & - \\
\hline 7 & 5 & 5 & $0.25 \mathrm{M}$ & 7 & - & - \\
\hline
\end{tabular}

${ }^{\mathrm{a}} \mathrm{wt} \%$ rel. to water

${ }^{\mathrm{b}} \mathrm{wt} \%$ rel. to monomer

${ }^{\mathrm{c}}$ Concentration rel. to monomer and HD

${ }^{\mathrm{d}} \mathrm{wt} \%$ rel. to organic phase

e $\mathrm{mol} \%$ rel. to acid

Table S3. Monomer conversion, intensity-average droplet diameter, polydispersity index (PdI), Mn and Mw data for $\mathrm{P}(\mathrm{St}-\mathrm{stat}-\mathrm{nBA}) / \mathrm{GO}$ synthesised via miniemulsion polymerization at $70{ }^{\circ} \mathrm{C}$ after $24 \mathrm{~h}$ with $5 \mathrm{wt} \% \mathrm{GO}$ (rel. to monomer) using different surfactants.

\begin{tabular}{|c|c|c|c|c|c|c|c|c|}
\hline \multirow{2}{*}{\multicolumn{2}{|c|}{$\mathrm{P}($ St-stat-nBA $) / \mathrm{GO}$}} & \multirow{3}{*}{$\begin{array}{c}\begin{array}{c}\text { Monomer } \\
\text { conversion } \\
(\%)\end{array} \\
96\end{array}$} & \multicolumn{2}{|c|}{ Droplet } & \multicolumn{2}{|c|}{ Particle } & \multirow{3}{*}{$\frac{\begin{array}{c}\mathrm{M}_{\mathrm{n}} \\
(\mathrm{kg} / \mathrm{mol})\end{array}}{139}$} & \multirow{3}{*}{$\frac{\begin{array}{c}\mathrm{M}_{\mathrm{w}} \\
(\mathrm{kg} / \mathrm{mol})\end{array}}{401}$} \\
\hline & & & \multirow{2}{*}{$\frac{\text { Size }(\mathrm{nm})}{516}$} & \multirow{2}{*}{$\frac{\mathrm{PdI}}{0.4}$} & \multirow{2}{*}{$\frac{\text { Size }(\mathrm{nm})}{75}$} & \multirow{2}{*}{$\frac{\mathrm{PdI}}{0.2}$} & & \\
\hline \multirow{4}{*}{ SDS } & $1 \mathrm{wt} \%$ & & & & & & & \\
\hline & $3 \mathrm{wt} \%$ & 100 & 221 & 0.6 & 72 & 0.4 & 170 & 515 \\
\hline & $5 \mathrm{wt} \%$ & 100 & 254 & 0.7 & 51 & 0.3 & 133 & 330 \\
\hline & $7 \mathrm{wt} \%$ & 100 & 260 & 0.7 & 59 & 0.2 & 150 & 371 \\
\hline \multirow{4}{*}{ KSA } & $1 \mathrm{wt} \%$ & 60 & 715 & 0.6 & - & - & 18 & 51 \\
\hline & $3 \mathrm{wt} \%$ & 93 & 224 & 0.4 & - & - & 192 & 805 \\
\hline & $5 \mathrm{wt} \%$ & 93 & 248 & 0.6 & - & - & 65 & 262 \\
\hline & $7 \mathrm{wt} \%$ & 96 & 303 & 0.8 & - & - & 125 & 570 \\
\hline \multirow{4}{*}{ KOA } & $1 \mathrm{wt} \%$ & 72 & 721 & 0.6 & - & - & 21 & 61 \\
\hline & $3 \mathrm{wt} \%$ & 78 & 283 & 0.5 & - & - & 53 & 236 \\
\hline & $5 \mathrm{wt} \%$ & 93 & 276 & 0.6 & - & - & 135 & 459 \\
\hline & $7 \mathrm{wt} \%$ & 97 & 415 & 0.7 & - & - & 115 & 380 \\
\hline
\end{tabular}

Table S4. Polymerization recipe for $\mathrm{P}(\mathrm{St}-$ stat- $n \mathrm{BA}) / \mathrm{GO}$ synthesized via miniemulsion polymerization at $70{ }^{\circ} \mathrm{C}$ after $24 \mathrm{~h}$ with $5 \mathrm{wt} \% \mathrm{GO}$ (rel. to monomer) using different conditions.

\begin{tabular}{|c|c|c|c|c|c|c|}
\hline \multirow{2}{*}{ Experiment } & \multirow{2}{*}{ Monomer $^{\mathrm{a}}$} & \multirow{2}{*}{$\mathrm{HD}^{\mathrm{b}}$} & \multirow{2}{*}{$\mathrm{GO}^{\mathrm{b}}$} & \multirow{2}{*}{$\mathrm{AIBN}^{\mathrm{c}}$} & \multicolumn{2}{|c|}{ In situ surfactant } \\
\cline { 5 - 7 } & & & & & Acid $^{\mathrm{d}}$ & Base $^{\mathrm{e}}$ \\
\hline $\mathrm{M} 1$ & 7 & 5 & 5 & $0.25 \mathrm{M}$ & $1 \mathrm{SA}$ & $2 \mathrm{KOH}$ \\
\hline M2 & 7 & 5 & 5 & $0.25 \mathrm{M}$ & $1 \mathrm{OA}$ & $2 \mathrm{KOH}$ \\
\hline M3 & 7 & 5 & 5 & $0.25 \mathrm{M}$ & $3 \mathrm{SA}$ & $2 \mathrm{NaOH}$ \\
\hline
\end{tabular}

${ }^{\mathrm{a}} \mathrm{wt} \%$ rel. to water

${ }^{\mathrm{b}} \mathrm{wt} \%$ rel. to monomer

${ }^{c}$ Concentration rel. to monomer and HD

${ }^{\mathrm{d}} \mathrm{wt} \%$ rel. to organic phase

${ }^{\mathrm{e}} \mathrm{mol} \%$ rel. to acid 
Table S5. Monomer conversion, intensity-average droplet diameter, polydispersity index (PdI), Mn and Mw data for $\mathrm{P}(\mathrm{St}-s t a t-n \mathrm{BA}) / \mathrm{GO}$ latex synthesised via miniemulsion polymerization at $70{ }^{\circ} \mathrm{C}$ after $24 \mathrm{~h}$ with $5 \mathrm{wt} \%$ $\mathrm{GO}$ (rel. to monomer) using different conditions.

\begin{tabular}{|c|c|c|c|c|c|}
\hline Experiment & $\begin{array}{c}\text { Monomer } \\
\text { conversion } \\
(\%)\end{array}$ & $\begin{array}{c}\text { Droplet } \\
\text { size (nm) }\end{array}$ & PdI & $\begin{array}{c}\mathrm{M}_{\mathrm{n}} \\
(\mathrm{kg} / \mathrm{mol})\end{array}$ & $\begin{array}{c}\mathrm{M}_{\mathrm{w}} \\
(\mathrm{kg} / \mathrm{mol})\end{array}$ \\
\hline $\mathrm{M} 1$ & 58 & 552 & 0.46 & 19 & 52 \\
\hline $\mathrm{M} 2$ & 74 & 389 & 0.56 & 20 & 48 \\
\hline $\mathrm{M} 4$ & 86 & 256 & 0.61 & 51 & 303 \\
\hline
\end{tabular}

Table S6. Raman data showing the $\mathrm{D}$ and $\mathrm{G}$ peaks positions and the $\mathrm{I}_{\mathrm{D}} / \mathrm{I}_{\mathrm{G}}$ ratios calculated from Lorentzian fitting of the deconvoluted $\mathrm{D}$ and $\mathrm{G}$ peaks obtained for thermally reduced $\mathrm{P}(\mathrm{St}-$ stat $-n \mathrm{BA}) / \mathrm{rGO}$ films containing different concentrations of surfactants.

\begin{tabular}{|c|c|c|c|}
\hline Surfactant & $\begin{array}{c}\text { D Band }\left(\mathrm{cm}^{-}\right. \\
1)\end{array}$ & $\begin{array}{c}\mathrm{G} \mathrm{Band}\left(\mathrm{cm}^{-}\right. \\
1)\end{array}$ & $\mathrm{I}_{\mathrm{D}} / \mathrm{I}_{\mathrm{G}}$ \\
\hline $1 \% \mathrm{SDS}$ & 1350.75 & 1587.20 & 0.81 \\
\hline $3 \%$ SDS & 1352.42 & 1587.14 & 0.75 \\
\hline $5 \%$ SDS & 1349.74 & 1584.83 & 0.75 \\
\hline $7 \%$ SDS & 1346.53 & 1583.15 & 0.81 \\
\hline $1 \%$ KSA & 1351.25 & 1586.27 & 0.84 \\
\hline $3 \%$ KSA & 1354.93 & 1587.12 & 0.83 \\
\hline $5 \%$ KSA & 1354.23 & 1586.45 & 0.77 \\
\hline $7 \%$ KSA & 1353.66 & 1586.81 & 0.8 \\
\hline $1 \%$ KOA & 1354.92 & 1586.32 & 0.76 \\
\hline $3 \%$ KOA & 1352.03 & 1587.62 & 0.75 \\
\hline $5 \%$ KOA & 1353.50 & 1586.54 & 0.83 \\
\hline $7 \%$ KOA & 1353.56 & 1586.80 & 0.83 \\
\hline
\end{tabular}

Table S7. Surface roughness $\left(\mathrm{R}_{\mathrm{a}}\right)$ of thermally reduced $\mathrm{P}(\mathrm{St}-$ stat- $n \mathrm{BA}) / \mathrm{rGO}(5 \mathrm{wt} \%)$ composite films at different concentrations of surfactants. Data are representative of two individual films and three measurements per film and presented as average \pm standard deviation.

\begin{tabular}{|c|c|c|}
\hline \multicolumn{2}{|c|}{ Surfactant } & $\mathrm{R}_{\mathrm{a}}(\mu \mathrm{m})$ \\
\hline \multirow{4}{*}{ SDS } & $1 \%$ & $0.98 \pm 0.08$ \\
\cline { 2 - 3 } & $3 \%$ & $0.60 \pm 0.07$ \\
\cline { 2 - 3 } & $5 \%$ & $1.86 \pm 0.34$ \\
\cline { 2 - 3 } & $7 \%$ & $0.92 \pm 0.22$ \\
\hline \multirow{4}{*}{ KSA } & $1 \%$ & $1.26 \pm 0.19$ \\
\cline { 2 - 3 } & $3 \%$ & $1.20 \pm 0.23$ \\
\cline { 2 - 3 } & $5 \%$ & $2.30 \pm 0.46$ \\
\cline { 2 - 3 } & $7 \%$ & $2.13 \pm 0.23$ \\
\hline \multirow{4}{*}{ KOA } & $1 \%$ & $1.25 \pm 0.3$ \\
\cline { 2 - 3 } & $3 \%$ & $1.41 \pm 0.18$ \\
\cline { 2 - 3 } & $5 \%$ & $1.31 \pm 0.21$ \\
\cline { 2 - 3 } & $7 \%$ & $1.27 \pm 0.20$ \\
\hline
\end{tabular}


Table S8. Electrical conductivity of thermally reduced P(St-stat- $n \mathrm{BA}) / \mathrm{rGO}$ composite films containing different surfactants at different concentrations. Data is representative of 2 individual films and 2 measurements per film and presented as average \pm standard deviation.

\begin{tabular}{|c|c|c|}
\hline \multicolumn{2}{|c|}{ Surfactant } & $\begin{array}{c}\text { Electrical conductivity } \\
(\mathrm{S} / \mathrm{m})\end{array}$ \\
\hline \multirow{4}{*}{$\mathrm{SDS}$} & $1 \mathrm{wt} \%$ & $0.6022 \pm 0.1329$ \\
\cline { 2 - 3 } & $3 \mathrm{wt} \%$ & $1.9477 \pm 0.0881$ \\
\cline { 2 - 3 } & $5 \mathrm{wt} \%$ & $2.3910 \pm 0.2546$ \\
\cline { 2 - 3 } & $7 \mathrm{wt} \%$ & $1.5756 \pm 0.1105$ \\
\hline \multirow{5}{*}{ KSA } & $1 \mathrm{wt} \%$ & $0.2253 \pm 0.0251$ \\
\cline { 2 - 3 } & $3 \mathrm{wt} \%$ & $0.0184 \pm 0.0025$ \\
\cline { 2 - 3 } & $5 \mathrm{wt} \%$ & $0.0034 \pm 0.0001$ \\
\cline { 2 - 3 } & $7 \mathrm{wt} \%$ & $0.0013 \pm 0.0001$ \\
\hline \multirow{5}{*}{ KOA } & $1 \mathrm{wt} \%$ & $0.3070 \pm 0.0543$ \\
\cline { 2 - 3 } & $3 \mathrm{wt} \%$ & $0.0141 \pm 0.0002$ \\
\cline { 2 - 3 } & $5 \mathrm{wt} \%$ & $0.0028 \pm 0.0002$ \\
\cline { 2 - 3 } & $7 \mathrm{wt} \%$ & $0.0011 \pm 0.0002$ \\
\hline
\end{tabular}

Table S9. Mechanical properties of $\mathrm{P}(\mathrm{St}-$ stat- $n \mathrm{BA}) / \mathrm{GO}$ composite films containing different surfactants (in situ KSA vs SDS) at different concentrations (3 $\mathrm{wt} \%, 5 \mathrm{wt} \%$ and $7 \mathrm{wt} \%$ ). Data are presented as an average \pm standard deviation of three independent films.

\begin{tabular}{|c|c|c|c|c|}
\hline \multicolumn{2}{|c|}{ Surfactant } & $\begin{array}{c}\text { Young's modulus } \\
(\mathrm{MPa})\end{array}$ & $\begin{array}{c}\text { Tensile strength } \\
(\mathrm{MPa})\end{array}$ & $\begin{array}{c}\text { Elongation at } \\
\text { break }(\%)\end{array}$ \\
\hline \multirow{3}{*}{$\mathrm{SDS}$} & $3 \mathrm{wt} \%$ & $102.33 \pm 29.69$ & $4.41 \pm 1.05$ & $433.66 \pm 115.94$ \\
\cline { 2 - 5 } & $5 \mathrm{wt} \%$ & $50.61 \pm 6.58$ & $3.87 \pm 0.83$ & $283.16 \pm 93.71$ \\
\cline { 2 - 5 } & $7 \mathrm{wt} \%$ & $58.17 \pm 10.94$ & $3.68 \pm 0.55$ & $256.76 \pm 85.47$ \\
\hline \multirow{3}{*}{ KSA } & $3 \mathrm{wt} \%$ & $60.02 \pm 8.89$ & $3.76 \pm 0.57$ & $60.34 \pm 14.45$ \\
\cline { 2 - 5 } & $5 \mathrm{wt} \%$ & $49.24 \pm 8.25$ & $3 \pm 0.22$ & $303.01 \pm 28.36$ \\
\cline { 2 - 5 } & $7 \mathrm{wt} \%$ & $64.73 \pm 29.14$ & $3.22 \pm 1.22$ & $386.38 \pm 92.93$ \\
\hline
\end{tabular}

Table S10. Mechanical properties of $\mathrm{P}(\mathrm{St}-\mathrm{stat}-n \mathrm{BA}) / \mathrm{GO}$ with $5 \mathrm{wt} \% \mathrm{GO}$ loading and neat polymer films containing $5 \mathrm{wt} \%$ in situ KSA. Data are presented as an average \pm standard deviation of three independent films.

\begin{tabular}{|c|c|c|c|c|}
\hline \multicolumn{2}{|c|}{ Polymer matrix } & $\begin{array}{c}\text { Young's modulus } \\
(\mathrm{MPa})\end{array}$ & $\begin{array}{c}\text { Tensile strength } \\
(\mathrm{MPa})\end{array}$ & $\begin{array}{c}\text { Elongation at break } \\
(\%)\end{array}$ \\
\hline \multirow{2}{*}{$\begin{array}{c}5 \mathrm{wt} \% \\
\mathrm{KSA}\end{array}$} & $\mathrm{P}(S t$-stat- $n \mathrm{BA}) / \mathrm{GO}$ & $49.24 \pm 8.25$ & $3 \pm 0.22$ & $303.01 \pm 28.36$ \\
\cline { 2 - 5 } & Neat polymer & $0.66 \pm 0.14$ & $0.24 \pm 0.05$ & $1307 \pm 62.49$ \\
\hline
\end{tabular}




\section{Supporting Information}

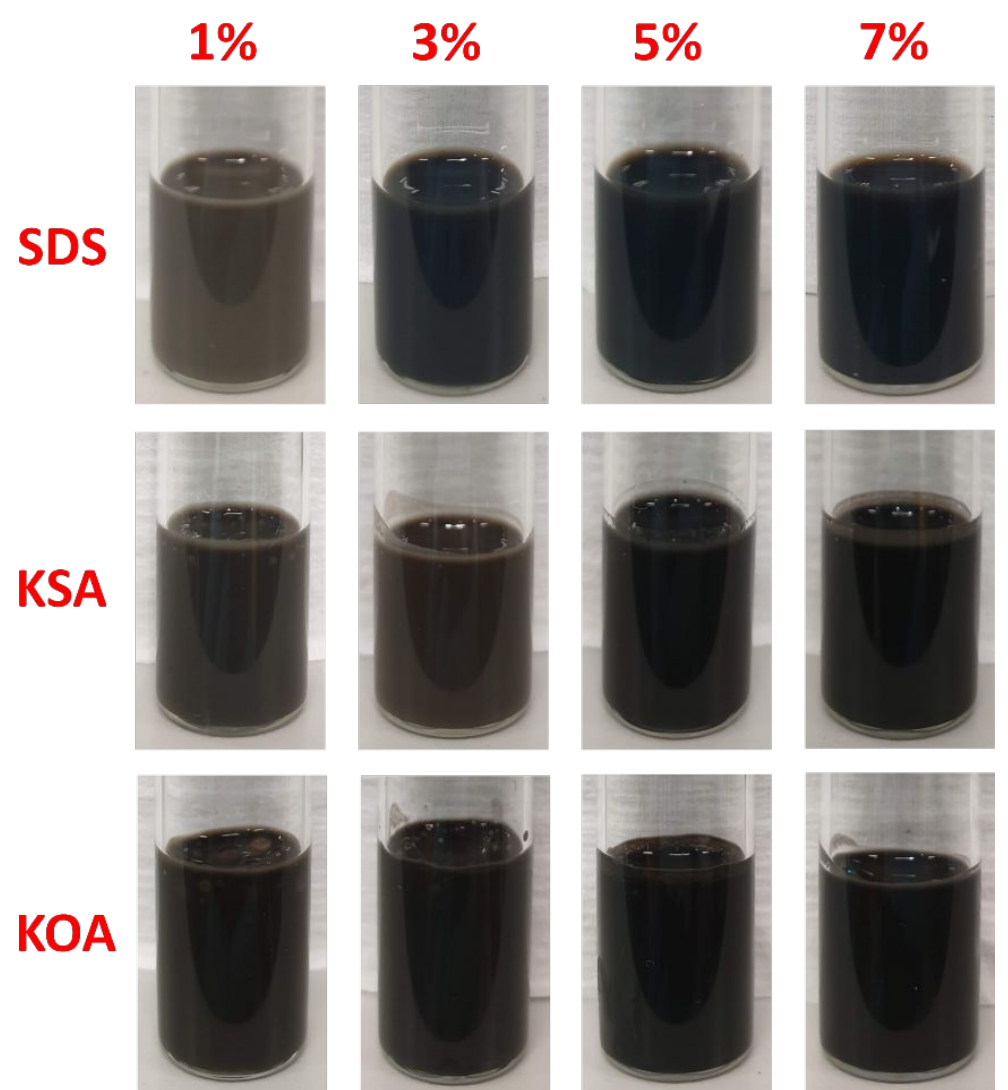

Figure S1. Digital photograph of the $\mathrm{P}(\mathrm{St}-\mathrm{stat}-n \mathrm{BA}) / \mathrm{GO}$ latexes containing different concentrations of surfactants synthesized via miniemulsion polymerization 
a)

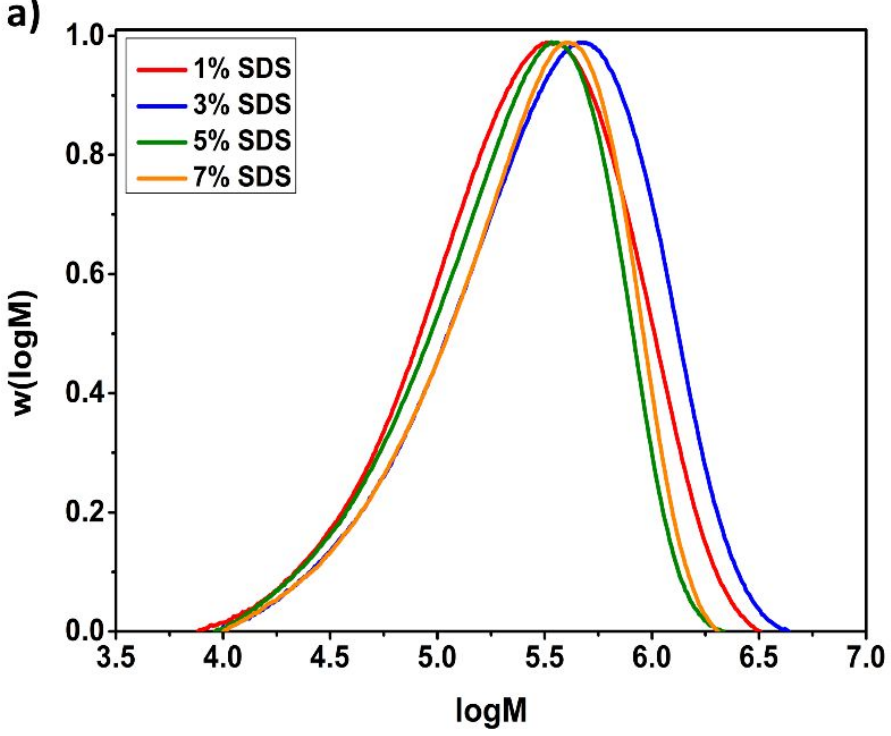

b)

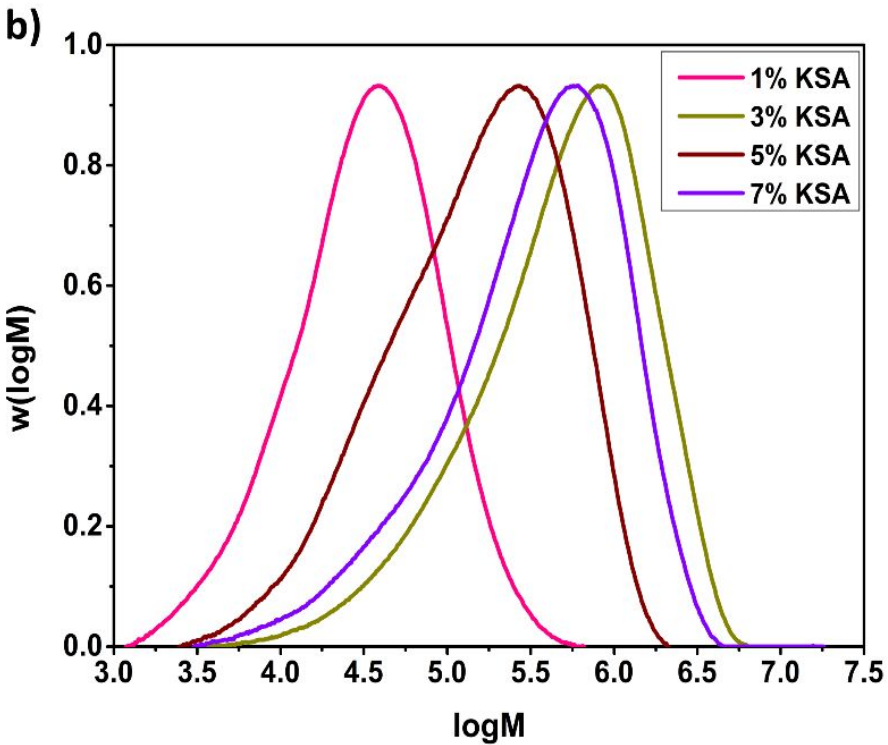

c)

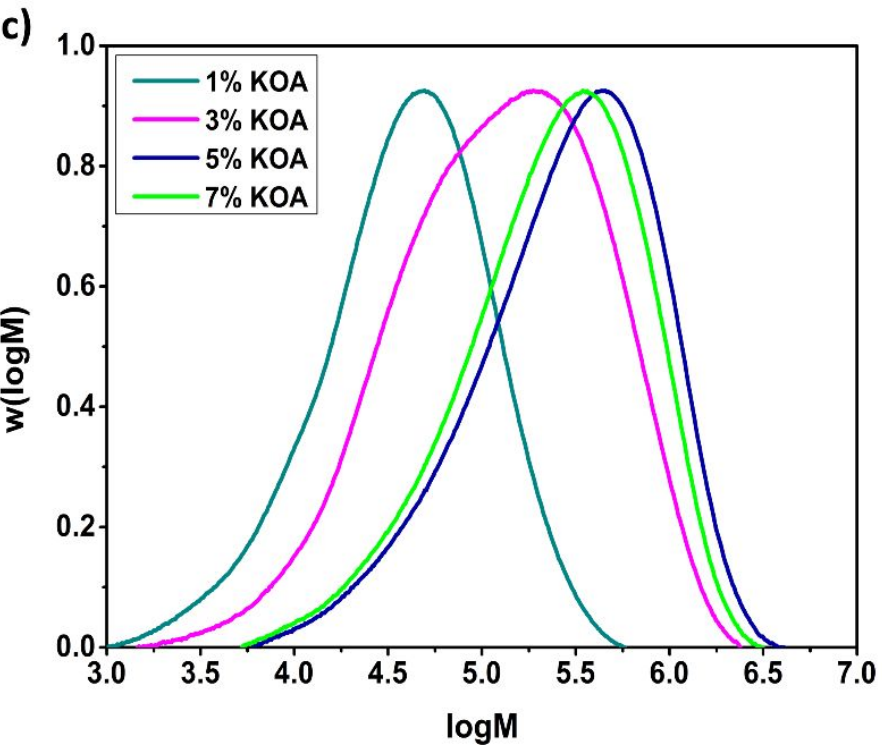

Figure S2. Molecular weight distributions for $\mathrm{P}(\mathrm{St}-$ stat- $n \mathrm{BA}) / \mathrm{GO}$ fabricated via miniemulsion polymerization at $70{ }^{\circ} \mathrm{C}$ after $24 \mathrm{~h}$ with $5 \mathrm{wt} \% \mathrm{GO}$ (rel. to monomer) using different surfactants. (a) SDS, (b) KSA and (c) KOA. 
a) SDS

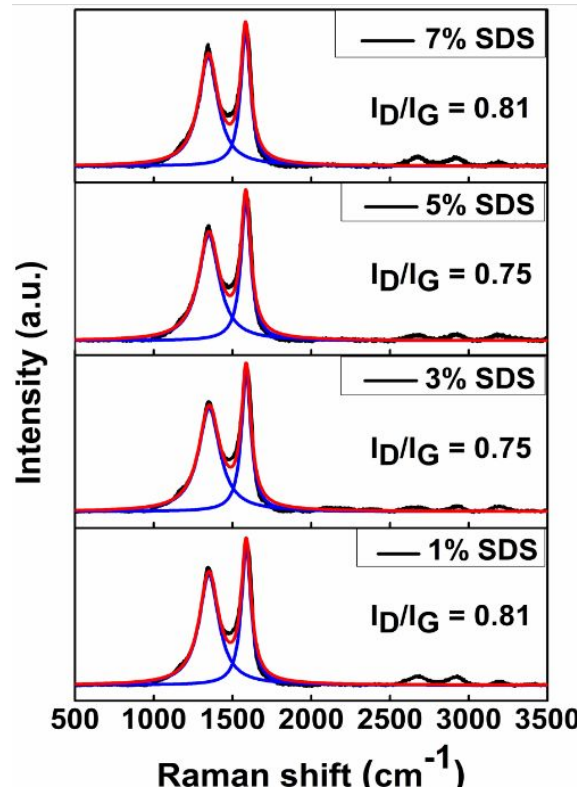

b) KSA

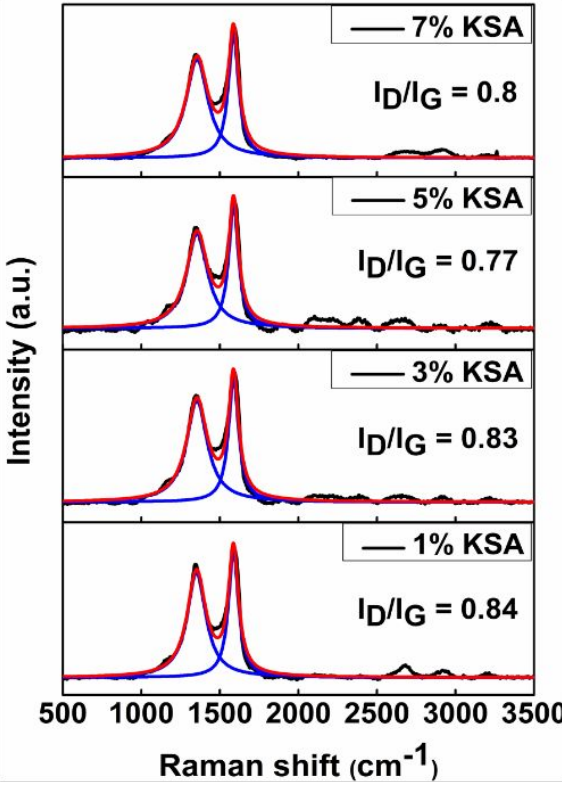

c) KOA

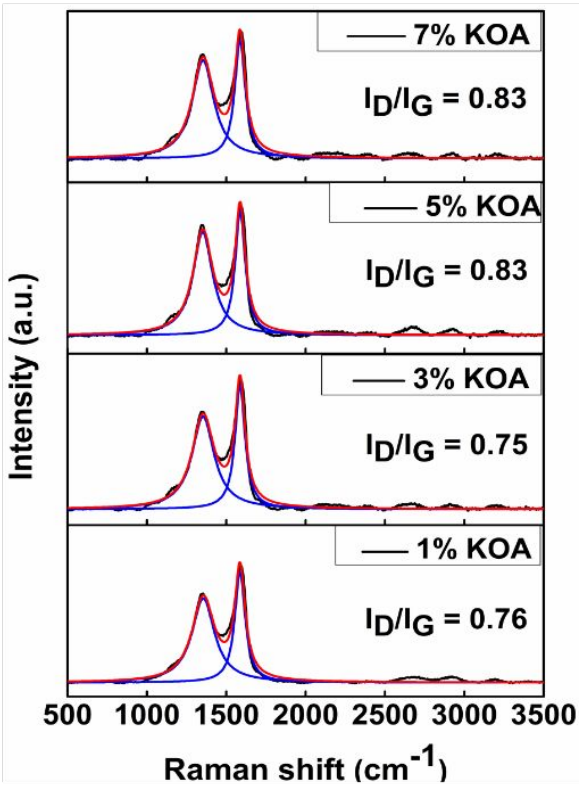

Figure S3. Raman analysis of $\mathrm{P}(\mathrm{St}-\mathrm{stat}-n \mathrm{BA}) / \mathrm{rGO}$ composite films containing different concentrations of (a) SDS, (b) KSA and (c) KOA.

\section{SDS}

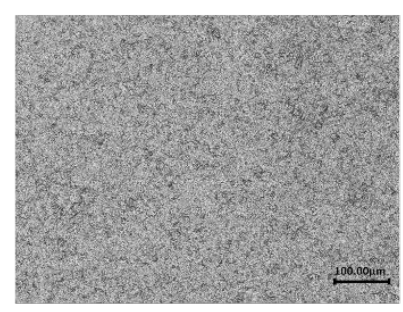

$3 \%$
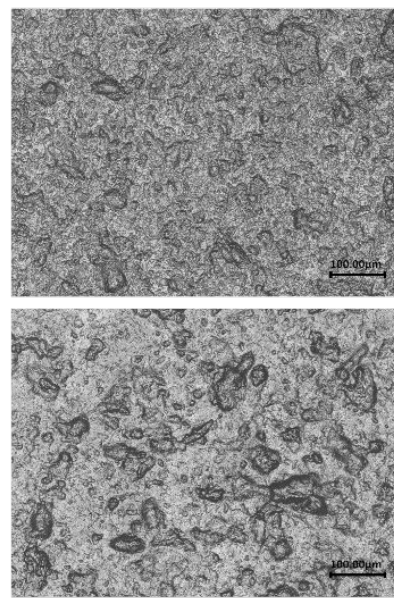

$5 \%$

$7 \%$

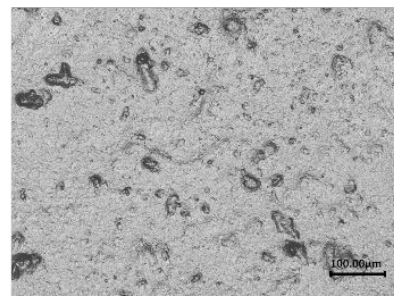

KSA
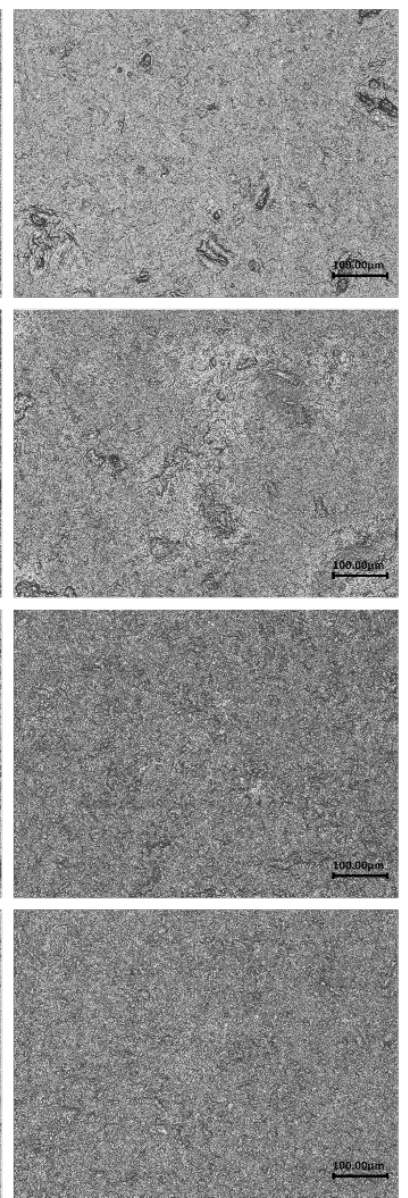

KOA
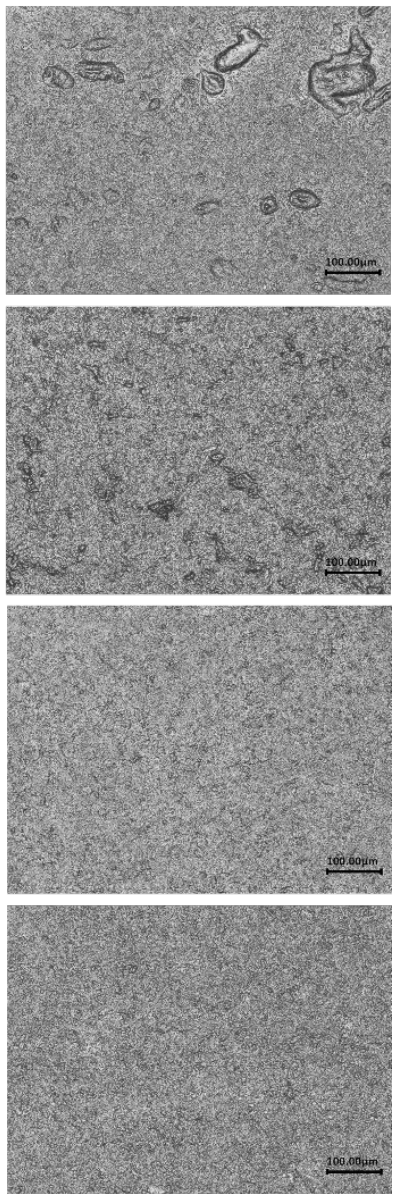

Figure S4. Additional confocal laser scanning microscopy images showing the surface morphology of the P(Ststat- $n \mathrm{BA}) / \mathrm{rGO}$ composite films fabricated using different concentrations of surfactants. Scale bar: $100 \mu \mathrm{m}$. 
Supporting Information

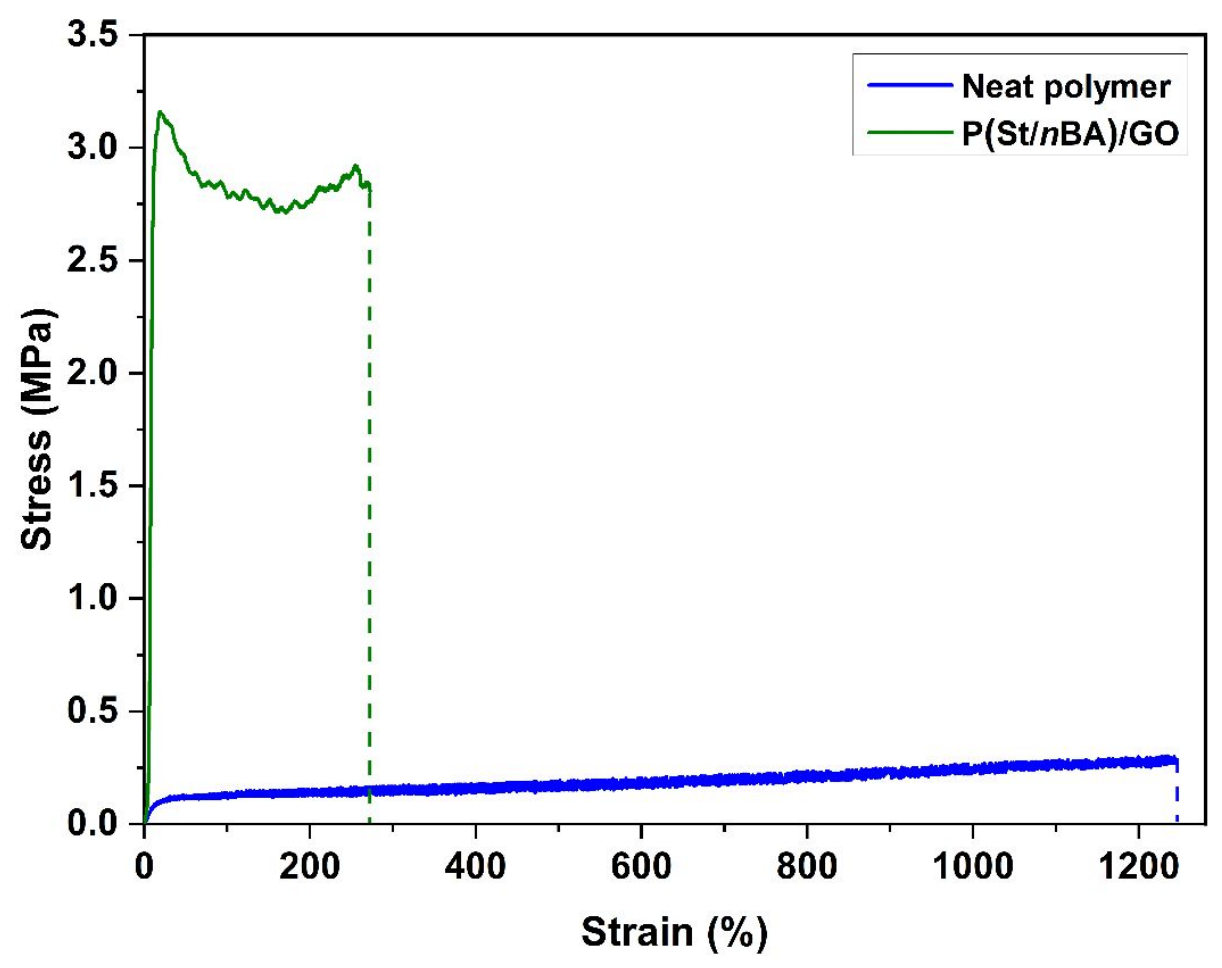

Figure S5. Stress-strain curve of $\mathrm{P}(\mathrm{St}-$ stat- $n \mathrm{BA}) / \mathrm{GO}$ with $5 \mathrm{wt} \%$ GO loading and neat polymer films containing $5 \mathrm{wt} \% \mathrm{KSA}$. 